\title{
Associations of serum calcium levels and dietary calcium intake with incident type 2 diabetes over 10 years: the Korean Genome and Epidemiology Study (KoGES)
}

\author{
Kyoung-Nam Kim ${ }^{1,2}$, Se-Young $\mathrm{Oh}^{3}$ and Yun-Chul Hong ${ }^{2,4,5^{*}}$
}

\begin{abstract}
Background: Previous evidence regarding the associations between serum calcium concentrations, dietary calcium intake, and type 2 diabetes (T2D) is limited. We investigated the longitudinal associations of serum calcium levels and dietary calcium intake with T2D development.

Methods: This study used data from the Ansung-Ansan cohort, a community-based, prospective cohort that was followed up for 10 years. Cox regression models adjusted for potential confounders were used to evaluate the associations of serum calcium levels (mean, $9.41 \mathrm{mg} / \mathrm{dL}$ ) and dietary calcium intake (median, $389.59 \mathrm{mg} /$ day) with T2D incidence. Association between dietary calcium intake and serum calcium levels was assessed using linear regression models.

Results: Albumin-adjusted serum calcium levels were not associated with T2D risk (hazard ratio $[H R]=1.07,95 \%$ confidence interval [CI] 0.96,1.19, $p$-value $=0.2333$ ). A one-unit increase in log-transformed, energy-adjusted dietary calcium intake was associated with a decreased risk of T2D $(H R=0.88,95 \% \mathrm{Cl} 0.77,1.00, p$-value $=0.0460)$ and lower albumin-adjusted serum calcium levels $(\beta=-0.04,95 \% \mathrm{Cl}-0.07,-0.02, p$-value $=0.0014)$. The associations did not differ according to sex (all $p$-values for interaction >0.10).

Conclusions: Serum calcium levels were not associated with T2D risk, while higher dietary calcium intake was associated with a decreased risk of T2D development. These results have public health implications for predicting and preventing T2D development, as well as providing guidelines for diet and calcium supplementation.
\end{abstract}

Keywords: Cohort study, Dietary calcium, Serum calcium, Type 2 diabetes mellitus

\section{Background}

Type 2 diabetes (T2D) is one of the most important public health issues worldwide, with the past few decades witnessing a rapid increase in the global number of affected patients [1]. Recently, limited number of studies have reported that higher serum calcium levels are associated with an increased risk of T2D development [2-5]. However, the association between serum calcium

\footnotetext{
*Correspondence: ychong1@snu.ac.kr

2 Department of Preventive Medicine, Seoul National University College of Medicine, 28 Yongon-Dong, Chongno-Gu, Seoul 110-799, Republic of Korea

Full list of author information is available at the end of the article
}

levels and T2D development was not observed in another epidemiological study [6]. In addition, the positive associations between serum calcium levels and T2D were unexpected, considering that higher calcium intake has been associated with lower T2D risk $[7,8]$.

Because the previous evidences on the associations between serum calcium concentrations, dietary calcium intake, and T2D development are inconsistent and limited, further investigation into these complex relationships are needed for predicting and preventing T2D development and providing scientific guidelines for diet and/or calcium supplementation in the general population. 
Therefore, in the present study, we evaluated the longitudinal associations of serum calcium levels and dietary calcium intake with incidence of T2D in a communitybased, prospective cohort, followed up for 10 years. We also assessed the association between dietary calcium intake and serum calcium levels at the baseline survey.

\section{Methods}

\section{Study design and population}

The present study was conducted using data from the Ansung-Ansan cohort, an ongoing communitybased, prospective cohort. Detailed information on the Ansung-Ansan cohort are presented elsewhere [9]. In brief, 10,038 participants aged 40-69 years who resided in the Ansung or Ansan regions of the Republic of Korea were recruited between 2001 and 2003 using a two-stage cluster sampling method. Follow-up surveys were conducted biennially. During each survey, participants took part in interviews using structured questionnaires, health examinations, and laboratory tests.

Among the 10,038 participants in the Ansung-Ansan cohort, 676 were excluded from analysis because they reported at enrollment that they had been previously diagnosed with T2D. In addition, 560 participants were excluded from analysis because they had prevalent T2D during the baseline survey, as determined by predefined criteria, based on fasting glucose concentration $(\geq 126 \mathrm{mg} / \mathrm{dL})$, post-load glucose concentration $(\geq 200 \mathrm{mg} / \mathrm{dL})$, and antidiabetic medication use. Two participants who lacked information on serum calcium levels were also excluded, resulting in the inclusion of 8800 participants in the final analysis. In summary, the exclusion criteria of the present study was presence of T2D and absence of serum calcium levels, and therefore, individuals with hypertension, dyslipidemia, or chronic kidney disease were included in the analysis. The present study used a sequential modeling approach and conducted several analyses with different covariate sets. Therefore, the number of samples used for each analysis was different between models, and this information, along with the results, is presented.

The Ansung-Ansan study protocol was reviewed and approved by the Institutional Review Board of the Korea Centers for Disease Control and Prevention, and all study participants submitted written informed consent. The present study was approved by the Ethical Review Board of Seoul National University Hospital (C-1306-046-495).

\section{Serum and dietary calcium assessment}

Serum calcium and albumin were enzymatically measured at the baseline survey using a 747 Chemistry Analyzer (Hitachi, Tokyo, Japan). Since total calcium levels may vary with serum albumin levels due to calcium-albumin binding, serum calcium concentrations were adjusted for albumin concentrations [2] using the following equation:

$$
\begin{aligned}
& \text { Albumin-adjusted serum calcium }(\mathrm{mg} / \mathrm{dL}) \\
& \begin{array}{c}
= \\
+ \\
+[0.8 \times(4-\text { albumin }(\mathrm{g} / \mathrm{dL}))] .
\end{array}
\end{aligned}
$$

The adjusted level was then used as an explanatory variable in the analysis.

Dietary data were collected during the baseline survey by trained interviewers using a validated semi-quantitative food frequency questionnaire [10]. The study participants reported the average consumption frequency and portion size of 103 food items during the previous years, and daily nutrient and total energy intake were calculated using the dietary habit information, as well as the nutrient and energy content for each food item. To control for potential confounding by energy intake, daily calcium intake was adjusted by total energy intake using the residual method [11]. Since the distribution of energy-adjusted calcium intake was highly skewed, logtransformed values were used in the analysis. The same analyses were also conducted using log-transformed calcium intake not adjusted for total energy intake, to assess the robustness of the results.

\section{Definition of T2D}

During each survey, a 75-g oral glucose tolerance test was performed for participants who were not known to have T2D and had not started diabetes treatment since the last survey. T2D was defined as any of the following conditions: fasting glucose concentration $\geq 126 \mathrm{mg} /$ $\mathrm{dL}$, post-load 2-h glucose concentration $\geq 200 \mathrm{mg} / \mathrm{dL}$, or antidiabetic medication use. In the present analysis, data for T2D detection was available for every survey from the baseline survey conducted in 2001-2003, up to the fifth follow-up survey conducted in 2011-2012.

\section{Statistical analysis}

The associations of albumin-adjusted serum calcium levels and energy-adjusted dietary calcium intake with T2D development were assessed using the Cox proportional hazards models with age as the time scale. The potential nonlinearity of the association was assessed through nonparametric analysis that applied spline smoothing methods [12].

We constructed analytical models by including covariates assessed at the baseline survey sequentially, to confirm robustness of the results. We selected the covariates based on previous literature and assumed biological pathways [2-5, 13]: (1) adjusted for age and sex; (2) further adjusted for residential area (Ansung or Ansan), 
monthly family income $(<\$ 869$, $\$ 869-1738, \$ 1738-$ 3475 , or $\geq \$ 3475$ ), tobacco smoking (non-smoker, exsmoker, current smoker), alcohol intake (non-drinker, ex-drinker, or current drinker), physical activity (none, $<3$, or $\geq 3$ episodes/week, with each episode defined as exercising for more than $30 \mathrm{~min}$ ), and body mass index $(<18.5,18.5-25,25-30$, or $\geq 30$; weight divided by height squared, $\mathrm{kg} / \mathrm{m}^{2}$ ); (3) further adjusted for systolic blood pressure $(\mathrm{mmHg})$, diastolic blood pressure $(\mathrm{mmHg})$, and serum creatinine level $(\mathrm{mg} / \mathrm{dL})$. Finally, we constructed the analytical model mutually adjusted for serum calcium levels and dietary calcium intake, by including terms for serum calcium levels, dietary calcium intake, and abovementioned covariates. To approximate the normal distribution, log-transformed values of dietary calcium intake and serum creatinine level were used in the analysis.

We assessed the cross-sectional association between dietary calcium intake and serum calcium levels at the baseline survey by linear regression models adjusted for the same covariate sets.

Potential heterogeneity of the associations of serum calcium levels and dietary calcium intake with T2D according to sex was explored because many of the previous studies only examined the association of calcium intake with T2D in women $[7,8,14]$. Interactions with sex were evaluated with the likelihood ratio test, by testing the product terms between sex and serum calcium levels or dietary calcium intake added to the main models.

We evaluated the robustness of the results by excluding those who had used diuretics or those who were using diuretics, because diuretics could also affect serum calcium levels.

All analyses were conducted using SAS version 9.4 (SAS Institute Inc., Cary, NC) and R version 3.2.5 (The Comprehensive $\mathrm{R}$ Archive Network, Vienna, Austria: http://cran.r-project.org).

\section{Results}

Baseline participant characteristics and associated serum calcium levels are presented in Table 1. The mean age at enrollment was 51.8 years, and there was a slightly higher proportion of women (53\%) than men (47\%). Majority of the participants were non-smokers (59\%), current drinkers (47\%), and did not exercise regularly (75\%). The mean body mass index was $24.5 \mathrm{~kg} / \mathrm{m}^{2}$. The mean (standard deviation) albumin-adjusted serum calcium level was $9.41(0.52) \mathrm{mg} / \mathrm{dL}$ (first quartile, $9.12 \mathrm{mg} / \mathrm{dL}$; median, $9.48 \mathrm{mg} / \mathrm{dL}$; third quartile, $9.76 \mathrm{mg} / \mathrm{dL}$ ). The median (interquartile range) dietary calcium intake was 389.59 (283.71) $\mathrm{mg} /$ day (first quartile, $268.94 \mathrm{mg} /$ day; third quartile, $552.65 \mathrm{mg} /$ day).
The associations of serum calcium levels and dietary calcium intake with T2D were found to be robust in penalized regression spline models (Fig. 1). In the fullyadjusted Cox proportional hazard models, we did not find the evidence for the association between serum calcium levels and risk of T2D (Table 2). However, a oneunit increase in log-transformed energy-adjusted dietary calcium intake was associated with a lower risk of T2D (Table 3). The associations between dietary calcium intake and T2D did not change appreciably between models not adjusted for serum calcium levels (hazard ratio $[\mathrm{HR}]=0.87,95 \%$ confidence interval $[\mathrm{CI}] 0.77,0.99)$ and models adjusted for serum calcium levels $(\mathrm{HR}=0.88$, $95 \%$ CI $0.77,1.00)$. These results were robust in analyses using dietary calcium not adjusted for total energy intake (data not shown).

When we assessed the association between dietary calcium intake and serum calcium levels at the baseline survey by linear regression model, dietary calcium intake was inversely associated with serum calcium levels after adjusting for potential confounders $(\beta=-0.04,95 \%$ CI $-0.07,-0.02$ ).

The associations of serum calcium levels and dietary calcium intake with T2D did not differ according to sex ( $p>0.10$ for all interactions).

After excluding those who had used diuretics (14 participants, $0.16 \%$ ) or those who were using diuretics at the baseline survey ( 8 participants, $0.09 \%$ ), the results were almost similar.

\section{Discussion}

In the present study with a community-based cohort followed-up for 10 years, the association between serum calcium levels and a risk of incident T2D was not evident, while higher dietary calcium intake was associated with a decreased risk of incident T2D.

Previous studies on the association between serum calcium levels and T2D have demonstrated inconsistent results. In a population-based prospective cohort study conducted in Norway, higher serum calcium concentrations were associated with an increased risk of incident T2D [3]. In a multicenter epidemiological study in the United States, in which participants were surveyed twice at an average of 5.2-year intervals, serum calcium levels from the first survey predicted an increased detection of T2D in the second survey [4]. In another prospective cohort study conducted among individuals with high cardiovascular risk in Spain, higher serum calcium levels were associated with T2D risk [2]. In a retrospective cohort study in China, elevated serum calcium levels were also associated with T2D risk [5]. However, in a prospective cohort study in Finland with a median follow-up of 23.1 years, levels of ionized calcium, a direct 
Table 1 Baseline characteristics and albumin-adjusted serum calcium concentrations of the study participants $(n=8800)$

\begin{tabular}{|c|c|c|c|}
\hline Characteristics & Values $^{\mathrm{a}}$ & Calcium $^{\mathbf{b}}$, mean (SD) & $p$-value \\
\hline Sex & & & $<0.0001$ \\
\hline Men & $4105(47)$ & $9.38(0.56)$ & \\
\hline Women & $4695(53)$ & $9.44(0.48)$ & \\
\hline Area & & & $<0.0001$ \\
\hline Ansung & $4358(50)$ & $9.44(0.49)$ & \\
\hline Ansan & $4442(50)$ & $9.38(0.54)$ & \\
\hline Monthly family income & & & $<0.0001$ \\
\hline$<\$ 869$ & $3000(35)$ & $9.48(0.49)$ & \\
\hline$\$ 869-\$ 1738$ & $2561(30)$ & $9.41(0.50)$ & \\
\hline$\$ 1738-\$ 3475$ & $2443(28)$ & $9.35(0.54)$ & \\
\hline$\geq \$ 3475$ & $634(7)$ & $9.24(0.56)$ & \\
\hline Tobacco smoking & & & $<0.0001$ \\
\hline Non-smoker & $5160(59)$ & $9.42(0.49)$ & \\
\hline Ex-smoker & $1305(15)$ & $9.34(0.56)$ & \\
\hline Current smoker & $2216(26)$ & $9.40(0.55)$ & \\
\hline Alcohol intake & & & $<0.0001$ \\
\hline Non-drinker & $4036(46)$ & $9.43(0.49)$ & \\
\hline Ex-drinker & $546(6)$ & $9.42(0.53)$ & \\
\hline Current drinker & $4137(47)$ & $9.38(0.54)$ & \\
\hline Physical activity & & & 0.0014 \\
\hline None & $6629(75)$ & $9.42(0.52)$ & \\
\hline$<3$ episodes/week & $428(5)$ & $9.35(0.53)$ & \\
\hline$\geq 3$ episodes/week & $1743(20)$ & $9.38(0.51)$ & \\
\hline Body mass index $\left(\mathrm{kg} / \mathrm{m}^{2}\right)$ & & & 0.0005 \\
\hline$<18.5$ & $167(2)$ & $9.42(0.51)$ & \\
\hline $18.5-25$ & $4997(57)$ & $9.39(0.52)$ & \\
\hline $25-30$ & $3225(37)$ & $9.43(0.52)$ & \\
\hline$\geq 30$ & $407(5)$ & $9.48(0.49)$ & \\
\hline Age (years) & $51.8(8.8)$ & & $<0.0001$ \\
\hline Systolic blood pressure $(\mathrm{mmHg})$ & $120.1(18.8)$ & & $<0.0001$ \\
\hline Diastolic blood pressure $(\mathrm{mmHg})$ & $79.6(12.1)$ & & 0.0073 \\
\hline Estimated glomerular filtration rate $\left(\mathrm{mL} / \mathrm{min} / 1.73 \mathrm{~m}^{2}\right)$ & $73.7(13.9)$ & & $<0.0001$ \\
\hline HOMA-IR & $1.3(1.9)$ & & 0.0049 \\
\hline Insulinogenic index & $0.4(3.9)$ & & 0.0247 \\
\hline Fasting glucose (mg/dL) & $83.0(8.8)$ & & $<0.0001$ \\
\hline Post-load 2-h glucose (mg/dL) & $115.3(30.9)$ & & 0.4335 \\
\hline $\mathrm{HbA1c}(\%)$ & $5.6(0.4)$ & & $<0.0001$ \\
\hline
\end{tabular}

SD standard deviation, HOMA-IR homeostatic model assessment-insulin resistance index, HbA1c glycated hemoglobin

a Values are presented as $n$ (\%) for categorical variables, mean (standard deviation) for age, systolic blood pressure, diastolic blood pressure, estimated glomerular filtration rate, fasting glucose level, post-load 2-h glucose level, and $\mathrm{Hb} 1 \mathrm{Ac}$ or geometric mean (geometric standard deviation) for the other continuous variables

b Albumin-adjusted serum calcium concentrations $(\mathrm{mg} / \mathrm{dL})$

c $p$-values are estimated by one-way analysis of variance for categorical variables and bivariate linear regression analysis for continuous variables

measurement of active serum calcium, were not associated with a T2D risk. The reason for the inconsistency in the results is not clear. However, differences in population characteristics such as race, genetic background, and vitamin $\mathrm{D}$, parathyroid, and phosphorus levels might be responsible for the inconsistency. In addition, difference in measurement errors in exposure and outcome and different covariates adjusted in analytical models might also contribute to the inconsistency. Because the number of studies exploring this association is limited, further studies, especially those using direct measure of active calcium, are warranted. 


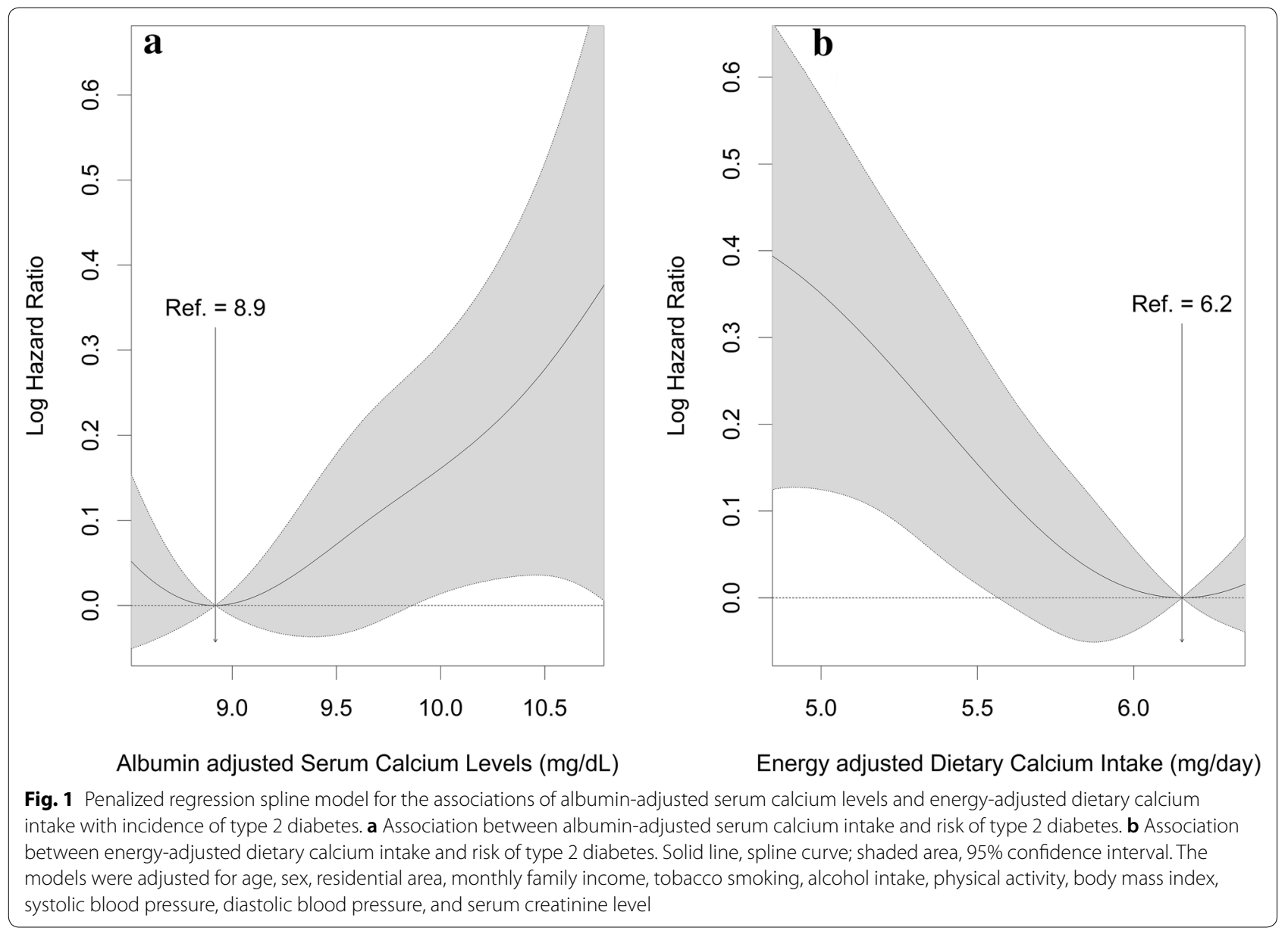

Table 2 Association between albumin-adjusted serum calcium levels and type 2 diabetes in the Ansung-Ansan cohort of the Korean Genome and Epidemiology Study (KoGES)

\begin{tabular}{lllll}
\hline & $\boldsymbol{n}^{\text {a }}$ & Hazard ratio & $\begin{array}{l}\text { 95\% } \\
\text { confidence } \\
\text { interval }\end{array}$ & -value \\
\hline Model 1 $^{\text {b }}$ & 8800 & 1.10 & $0.99,1.22$ & 0.0919 \\
Model 2 $^{c}$ & 8534 & 1.10 & $0.98,1.22$ & 0.0947 \\
Model 3 $^{\mathrm{d}}$ & 8525 & 1.07 & $0.96,1.19$ & 0.2125 \\
Model 4 $^{\mathrm{e}}$ & 8433 & 1.07 & $0.96,1.19$ & 0.2333 \\
\hline
\end{tabular}

a Number of study participants included in each analysis

b Adjusted for age and sex

c Adjusted as in Model 1, and further adjusted for residential area, monthly family income, tobacco smoking, alcohol intake, physical activity, and body mass index

d Adjusted as in Model 2, and further adjusted for systolic blood pressure, diastolic blood pressure, and serum creatinine level

e Adjusted as in Model 3, and further adjusted for dietary calcium intake
Four large cohort studies have investigated the association between calcium intake and T2D in the United States $(n=83,779$ [7], $(n=41,186)$ [14]), China $(n=64,191)$ [8], and Japan $(n=59,796)$ [15]. These studies reported inverse associations between dietary or total calcium intake and T2D risk among women in the United States, women in China, or individuals with higher vitamin D intake in Japan. Another cohort study conducted in rural areas in Korea $(n=8313)$ also reported an inverse association between total and vegetable calcium intake, and T2D risk among women [16]. However, a relatively small study $(n=5200)$ in Australia found no association between dietary calcium and T2D [17]. Additionally, studies provided mixed results when investigating the association between dairy products (a key dietary calcium source) and T2D. One confounder could be the high fat content in some dairy products, which may mitigate the protective effects of calcium $[8,18]$. Calcium 
Table 3 Association between dietary calcium intake and type 2 diabetes in the Ansung-Ansan cohort of the Korean Genome and Epidemiology Study (KoGES)

\begin{tabular}{lllll}
\hline & $\boldsymbol{n}^{\mathbf{a}}$ & Hazard ratio & $\begin{array}{l}\mathbf{9 5 \%} \\
\text { confidence } \\
\text { interval }\end{array}$ & $\boldsymbol{p}$-value \\
\hline Model 1 $^{\mathrm{b}}$ & 8685 & 0.90 & $0.80,1.02$ & 0.0900 \\
Model 2 $^{\mathrm{c}}$ & 8444 & 0.87 & $0.77,0.99$ & 0.0359 \\
Model 3 $^{\mathrm{d}}$ & 8433 & 0.87 & $0.77,0.99$ & 0.0390 \\
Model 4 $^{\mathrm{e}}$ & 8433 & 0.88 & $0.77,1.00$ & 0.0460 \\
\hline
\end{tabular}

${ }^{a}$ Number of study participants included in each analysis

b Adjusted for age and sex

c Adjusted as in Model 1, and further adjusted for residential area, monthly family income, tobacco smoking, alcohol intake, physical activity, and body mass index

d Adjusted as in Model 2, and further adjusted for systolic blood pressure, diastolic blood pressure, and serum creatinine level

e Adjusted as in Model 3, and further adjusted for serum calcium level

intake may also depend on non-dairy foods, including tofu, fish, rice, vegetables, and legumes, so the main source of dietary calcium may differ across populations and cultures [8]. Thus, geographically diverse studies may help to evaluate the association between calcium intake and T2D by reducing the effects of this potential confounder. However, possibility that the observed inverse association between dietary calcium intake and T2D risk might be attributable to dietary source of calcium still remains.

In the present study, serum calcium levels were not associated with T2D risk, while dietary calcium intake was inversely associated with T2D risk. This might be because serum calcium levels could reflect not only exogenous calcium intake but also the ability to maintain homeostasis.

Dietary calcium intake was inversely associated with serum calcium levels. Although vitamin D insufficiency is highly prevalent in the Republic of Korea [19] and lower calcium intake was reported to be associated with an increased parathyroid hormone levels in individuals with low serum vitamin D levels [20], to our knowledge, there has been few study directly investigating these inverse association and underlying mechanisms. Further studies using the information on vitamin $\mathrm{D}$ or parathyroid hormone are warranted.

Calcium reportedly functions in intracellular processes mediated by insulin in skeletal muscle and adipose tissue, and potentially affects insulin sensitivity in these insulinresponsive tissues $[21,22]$. Calcium is also essential for insulin secretion from pancreatic $\beta$-cells in response to elevated blood glucose levels, acting via voltage-gated calcium channels or large transient receptor potential channels [23]. Increased calcium intake may also decrease the risk of osteoporosis and reduce the release of environmental pollutants such as lead, which are sequestered in bone and can increase insulin resistance and T2D risk [24]. In addition, an inverse association between dietary calcium intake and a risk of T2D may be indirectly attributable to changes in gastrointestinal hormones or intestinal microbiota and integrity [25].

There are several limitations of the present study. First, although ionized calcium is physiologically active and a measurable biomarker of calcium homeostasis, the pertinent data were not available. Instead, the present study used albumin-adjusted serum calcium levels, which is highly correlated with ionized calcium with large sample sizes [26], to consider variation in total calcium levels according to serum albumin $[2,4,5]$. Second, although vitamin $\mathrm{D}$ or parathyroid hormone may affect the association between serum calcium levels and T2D, we had no information available regarding these biomarkers. This limited our ability to comprehensively evaluate the associations between calcium, vitamin D, parathyroid hormone, and T2D. Third, calcium supplement information was lacking, which could result in misclassification of total exogenous calcium intake.

However, the present study also has considerable strengths. The study used data from a well-designed, community-based prospective cohort that was followed up for 10 years. The data included various clinical, dietary, and epidemiological traits; they provided a unique opportunity to investigate the complex association between serum calcium levels, dietary calcium intake, and incident T2D.

\section{Conclusions}

Serum calcium levels were not associated with a T2D risk, but higher dietary calcium intake was associated with a decreased T2D risk. The present results have potential public health implications for predicting and preventing T2D development, as well as providing guidelines for diet and calcium supplementation. Because previous evidence regarding the association between serum calcium levels, dietary calcium intake, and T2D are insufficient, further studies conducted among different populations are warranted to confirm the present results.

\section{Abbreviations \\ $\mathrm{Cl}$ : confidence interval; HR: hazard ratio; T2D: type 2 diabetes.}

\section{Authors' contributions}

KNK and YCH developed the study concept and design. KNK, SYO, and YCH analyzed and interpreted data, and drafted the manuscript. KNK performed the statistical analysis. KNK had full access to all data in the study and takes responsibility for the integrity of the data and the accuracy of the data analysis. All authors read and approved the final manuscript. 


\begin{abstract}
Author details
${ }^{1}$ Division of Public Health and Preventive Medicine, Seoul National University Hospital, 101 Daehak-Ro Jongno-Gu, Seoul, Republic of Korea. ${ }^{2}$ Department of Preventive Medicine, Seoul National University College of Medicine, 28 Yongon-Dong, Chongno-Gu, Seoul 110-799, Republic of Korea. ${ }^{3}$ Department of Food and Nutrition, Research Center for Human Ecology, College of Human Ecology, Kyung Hee University, 26 Kyungheedae-Ro Dongdaemun-Gu, Seoul, Republic of Korea. ${ }^{4}$ Institute of Environmental Medicine, Seoul National University Medical Research Center, 103 Daehak-Ro Jongno-Gu, Seoul, Republic of Korea. ${ }^{5}$ Environmental Health Center, Seoul National University College of Medicine, 103 Daehak-Ro Jongno-Gu, Seoul, Republic of Korea.
\end{abstract}

\section{Acknowledgements}

We thank the participants and survey staff of the Korean Genome and Epidemiology Study (KoGES) for their contributions to the present study.

\section{Competing interests}

The authors declare they have no competing interests.

\section{Availability of data and materials}

The dataset used in this study (Ansung-Ansan cohort) can be provided after review and evaluation of research plan by the Korea Centers for Disease Control and Prevention (http://www.cdc.go.kr/CDC/eng/main.jsp).

\section{Consent for publication \\ Not applicable.}

\section{Ethics approval and consent to participate}

The Ansung-Ansan study protocol was reviewed and approved by the Institutional Review Board of the Korea Centers for Disease Control and Prevention, and all study participants submitted written informed consent. The present study was approved by the Ethical Review Board of Seoul National University Hospital (C-1306-046-495).

\section{Funding}

This study was supported by grants from the Korea Centers for Disease Control, Republic of Korea (4845-301, 4851-302, and 4851-307). This study was also supported in part by the R\&D Program for Society of the National Research Foundation funded by the Ministry of Science, ICT \& Future Planning, Republic of Korea (2014M3C8A5030619). The sponsors had no role in the design and conduct of the study; collection, management, analysis, and interpretation of the data; preparation, review, or approval of the manuscript; or decision to submit the manuscript for publication.

\section{Publisher's Note}

Springer Nature remains neutral with regard to jurisdictional claims in published maps and institutional affiliations.

Received: 2 April 2018 Accepted: 2 June 2018

Published online: 19 June 2018

\section{References}

1. Danaei G, Finucane MM, Lu Y, Singh GM, Cowan MJ, Paciorek CJ, et al. National, regional, and global trends in fasting plasma glucose and diabetes prevalence since 1980: systematic analysis of health examination surveys and epidemiological studies with 370 country-years and 2.7 million participants. Lancet. 2011;378:31-40.

2. Becerra-Tomás N, Estruch R, Bulló M, Casas R, Díaz-López A, Basora J, et al. Increased serum calcium levels and risk of type 2 diabetes in individuals at high cardiovascular risk. Diabetes Care. 2014;37:3084-91.

3. Jorde R, Schirmer H, Njølstad I, Løchen M-L, Bøgeberg Mathiesen E, Kamycheva E, et al. Serum calcium and the calcium-sensing receptor polymorphism rs17251221 in relation to coronary heart disease, type 2 diabetes, cancer and mortality: the Troms $\varnothing$ Study. Eur J Epidemiol. 2013;28:569-78.

4. Lorenzo C, Hanley AJ, Rewers MJ, Haffner SM. Calcium and phosphate concentrations and future development of type 2 diabetes: the insulin resistance atherosclerosis study. Diabetologia. 2014;57:1366-74.
5. Sing CW, Cheng VKF, Ho DKC, Kung AWC, Cheung BMY, Wong ICK, et al. Serum calcium and incident diabetes: an observational study and metaanalysis. Osteoporos Int. 2016;27:1747-54

6. Zaccardi F, Webb DR, Carter P, Pitocco D, Khunti K, Davies MJ, et al. Association between direct measurement of active serum calcium and risk of type 2 diabetes mellitus: a prospective study. Nutr Metab Cardiovasc Dis. 2015;25:562-8.

7. Pittas AG, Dawson-Hughes B, Li T, Van Dam RM, Willett WC, Manson $\mathrm{JE}$, et al. Vitamin $\mathrm{D}$ and calcium intake in relation to type 2 diabetes in women. Diabetes Care. 2006;29:650-6.

8. Villegas R, Gao Y-T, Dai Q, Yang G, Cai H, Li H, et al. Dietary calcium and magnesium intakes and the risk of type 2 diabetes: the Shanghai Women's Health Study. Am J Clin Nutr. 2009;89:1059-67.

9. Shin C, Abbott RD, Lee H, Kim J, Kimm K. Prevalence and correlates of orthostatic hypotension in middle-aged men and women in Korea: the Korean Health and Genome Study. J Hum Hypertens. 2004;18:717-23.

10. Ahn Y, Kwon E, Shim JE, Park MK, Joo Y, Kimm K, et al. Validation and reproducibility of food frequency questionnaire for Korean genome epidemiologic study. Eur J Clin Nutr. 2007;61:1435-41.

11. Hu FB, Stampfer MJ, Rimm E, Ascherio A, Rosner BA, Spiegelman D, et al. Dietary fat and coronary heart disease: a comparison of approaches for adjusting for total energy intake and modeling repeated dietary measurements. Am J Epidemiol. 1999;149:531-40.

12. Meira-Machado L, Cadarso-Suárez C, Gude F, Araújo A. smoothHR: an R package for pointwise nonparametric estimation of hazard ratio curves of continuous predictors. Comput Math Methods Med. 2013;2013:745742.

13. Cho NH, Kim KM, Choi SH, Park KS, Jang HC, Kim SS, et al. High blood pressure and its association with incident diabetes over 10 years in the Korean Genome and Epidemiology Study (KoGES). Diabetes Care. 2015;38:1333-8.

14. van Dam RM, Hu FB, Rosenberg L, Krishnan S, Palmer JR. Dietary calcium and magnesium, major food sources, and risk of type 2 diabetes in U.S. black women. Diabetes Care. 2006;29:2238-43.

15. Kirii K, Mizoue T, Iso H, Takahashi Y, Kato M, Inoue M, et al. Calcium, vitamin $\mathrm{D}$ and dairy intake in relation to type 2 diabetes risk in a Japanese cohort. Diabetologia. 2009;52:2542-50.

16. Oh JM, Woo HW, Kim MK, Lee Y-H, Shin DH, Shin M-H, et al. Dietary total, animal, vegetable calcium and type 2 diabetes incidence among Korean adults: the Korean Multi-Rural Communities Cohort (MRCohort). Nutr Metab Cardiovasc Dis. 2017;27:1152-64.

17. Gagnon C, Lu ZX, Magliano DJ, Dunstan DW, Shaw JE, Zimmet PZ, et al. Serum 25-hydroxyvitamin D, calcium intake, and risk of type 2 diabetes after 5 years: results from a national, population-based prospective study (the Australian Diabetes, Obesity and Lifestyle study). Diabetes Care. 2011;34:1133-8.

18. Aune D, Norat T, Romundstad P, Vatten LJ. Dairy products and the risk of type 2 diabetes: a systematic review and dose-response meta-analysis of cohort studies. Am J Clin Nutr. 2013;98:1066-83.

19. Choi HS, Oh HJ, Choi H, Choi WH, Kim JG, Kim KM, et al. Vitamin D insufficiency in Korea - a greater threat to younger generation: the Korea National Health and Nutrition Examination Survey (KNHANES) 2008. J Clin Endocrinol Metab. 2011;96:643-51.

20. Steingrimsdottir L, Gunnarsson O, Indridason OS, Franzson L, Sigurdsson G. Relationship between serum parathyroid hormone levels, vitamin D sufficiency, and calcium intake. JAMA. 2005;294:2336-41.

21. Wright DC, Hucker KA, Holloszy JO, Han DH. Ca2+ and AMPK both mediate stimulation of glucose transport by muscle contractions. Diabetes. 2004;53:330-5.

22. Zemel MB. Nutritional and endocrine modulation of intracellular calcium: implications in obesity, insulin resistance and hypertension. Mol Cell Biochem. 1998:188:129-36.

23. Cheng H, Beck A, Launay P, Gross SA, Stokes AJ, Kinet J-P, et al. TRPM4 controls insulin secretion in pancreatic beta-cells. Cell Calcium. 2007;41:51-61.

24. Hong H, Kim E-K, Lee J-S. Effects of calcium intake, milk and dairy product intake, and blood vitamin D level on osteoporosis risk in Korean adults: analysis of the 2008 and 2009 Korea National Health and Nutrition Examination Survey. Nutr. Res. Pract. 2013;7:409-17.

25. Gomes JMG, Costa JA, Alfenas RC. Could the beneficial effects of dietary calcium on obesity and diabetes control be mediated by changes in intestinal microbiota and integrity? Br J Nutr. 2015;114:1756-65.

26. Baird GS. Ionized calcium. Clin Chim Acta. 2011:412:696-701. 